\title{
Validation of the face-name pairs task in major depression: impaired recall but not recognition
}

\author{
Kimberley J. Smith ${ }^{1}$, Sinead Mullally' , Declan McLoughlin ${ }^{2}$ and Shane O'Mara' * \\ 'Trinity College Institute of Neuroscience, Trinity College Dublin, Dublin, Ireland \\ ${ }^{2}$ Neurobiology of Depression Research Group, St. Patrick's Hospital, Dublin, Ireland
}

\section{Edited by:}

Xavier Noel, Université Libre de

Bruxelles, Belgium

\section{Reviewed by:}

Pierre Maurage, University of Louvain, Belgium

Sherry Stewart, Dalhousie University,

Canada

\section{${ }^{*}$ Correspondence:}

Shane O'Mara, Trinity College

Institute of Neuroscience, Lloyd

Building, Trinity College Dublin,

Dublin 2, Ireland

e-mail:smomara@tcd.ie
Major depression can be associated with neurocognitive deficits which are believed in part to be related to medial temporal lobe pathology. The purpose of this study was to investigate this impairment using a hippocampal-dependent neuropsychological task. The face-name pairs task was used to assess associative memory functioning in 19 patients with major depression. When compared to age-sex-and-education matched controls, patients with depression showed impaired learning, delayed cued-recall, and delayed freerecall. However, they also showed preserved recognition of the verbal and nonverbal components of this task. Results indicate that the face-name pairs task is sensitive to neurocognitive deficits in major depression.

Keywords: depression, recall, recognition, cognition, memory, face-name pairs

\section{INTRODUCTION}

Major depressive disorder (MDD) is one of the leading causes of disability worldwide, with an estimated 350 million people affected (World Health Organisation, 2012). Some of the most occupationally debilitating symptoms of depression are the widespread neurocognitive deficits (Mackin and Arean, 2009; Baune etal., 2010) that often accompany the core pathological symptoms of depressed mood and anhedonia (DSM-IV-TR, 2004). These cognitive impairments include deficits in working memory, attention, verbal fluency, executive functioning, and long-term memory (Landro et al., 2001; Rogers et al., 2004; Baune et al., 2010; Hammar and Ardal, 2009; Reppermund et al., 2009).

Long-term memory impairments are in part characterized by poorer delayed recall of both verbal and nonverbal items (Reischies and Neu, 2000; Landro et al., 2001; Naismith et al., 2003; Vythilingam et al., 2004). As well as showing poorer recall, some studies also demonstrate depressed patients to have impaired recognition with preserved familiarity (MacQueen etal., 2002; Ramponi et al., 2004; Drakeford et al., 2010). This memory impairment is believed to be the result of hippocampal pathology as depressed patients show reduced hippocampal volumes (Sheline et al., 1996, 1999; Mervaala et al., 2000; Bremner et al., 2004; Frodl etal., 2006) that can be correlated with the degree of memory impairment (Hickie et al., 2005).

The face-name pairs task (FNP) is a widely regarded paradigm of associative memory which is associated with activation of the anterior hippocampus (Sperling etal., 2003; Zeineh et al., 2003; Chua etal., 2007). This task has been previously used and shown to have sensitivity in various clinical and psychiatric populations including people with bipolar disorder (Glahn et al., 2010) and Alzheimer's disease (van Paasschen et al., 2013).
However to our knowledge, this test has never been used in patients with a MDD. The aim of this study was to assess whether the face-name pairs task was sensitive to 1 . Differences in recall and recognition in a depressed population and 2. Differences between a Depressed group and a Control group.

\section{METHODS}

Nineteen in-patients MDD were recruited from St. Patrick's Hospital Dublin and compared to 17 age-education-and-sex-matched control participants.

Depressed patients were identified with the help of nursing staff, and by assessing patient charts for current psychiatric diagnosis Potential participants were then approached directly to ask whether they were willing to take part. Inclusion criteria included being between the ages of 18-65 and having MDD of mild to moderate severity (as indicated by a severity of $\geq 8$ on the Hamilton Depression Inventory). Presence of current MDD (past month) was confirmed by completion of the Structured Clinical Interview for DSM-IV disorders prior to study commencement. Exclusion criteria included any other significant medical or psychiatric comorbidity, or being on medication such as antipsychotics or which could adversely influence results (see Table 1 for demographic information).

Control participants were recruited via the Trinity College Dublin electronic notice board and an advertisement in the Irish Times newspaper. Inclusion criteria included being between 18 and 65 years of age, and exclusion criteria included having any significant medical co-morbidities, being pregnant, or current psychiatric morbidity (as confirmed by completion of the structured clinical interview). This study was carried out with ethical approval from the psychology ethics counsel of Trinity College Dublin and 
Table 1 | Table showing demographic information for the participants in this study.

\begin{tabular}{lllll}
\hline Group & $\boldsymbol{N}$ & Age & Gender & Education \\
\hline Control & 17 & $46.24 \pm 3.2$ & $\begin{array}{l}\text { Male: 11 }(65 \%) ; \\
\text { female: 6 (35\%) }\end{array}$ & $14.5 \pm 0.5$ \\
Depressed & 19 & $39.32 \pm 2.8$ & $\begin{array}{l}\text { Male: 13 (68\%); } \\
\text { female: 6 (32\%) }\end{array}$ & $13.3 \pm 0.4$ \\
& & & & \\
\hline
\end{tabular}

There was no significant difference between the groups when assessed using an independent-samples t-test for age [t(34) $=0.52, P=0.1]$, or education $[t(34)=0.93, P=0.4]$. When gender was assessed using a chi-squared anal$y$ sis, there was no significant difference between groups for the percentages of males and females $\left[x^{2}(1, N=35)=0.23, P=0.6\right]$.

the ethics board at St. Patrick's Hospital. All participants gave their full informed consent prior to the commencement of testing.

\section{MATERIALS AND METHODS}

Participants were tested using the 28-item Everyday Memory Questionnaire (EMQ) and a computerized version of the FNP task as described below.

\section{FACE-NAME PAIRS TASK}

\section{Materials and stimuli}

This face-name task is a modified version of the design described by Zeineh et al. (2003). In order to avoid potential problems with "floor" results, due to the older age of the population studied, the number of faces to be remembered was reduced from eight to six. The six faces presented were all female (selected from a college yearbook, all presented in black and white and with all their hair removed). During both the encoding and retrieval phases these faces were presented on the right half of the screen. In the encoding phase the left side of the screen would contain the corresponding name, and in the retrieval phase the name was replaced by the prompt "Name?” (see Figure 1).

\section{Procedure}

This task comprised four blocks of immediate recall, where participants were presented with six face-name pairs (see Figure 1B) followed by a distracter task (see Figure 1B) and finally retrieval, where participants were prompted to vocally recall the name of each face (see Figure 1B). Following a half hour break one block of delayed recall with face and name recognition components where participants were required to identify

\section{A}

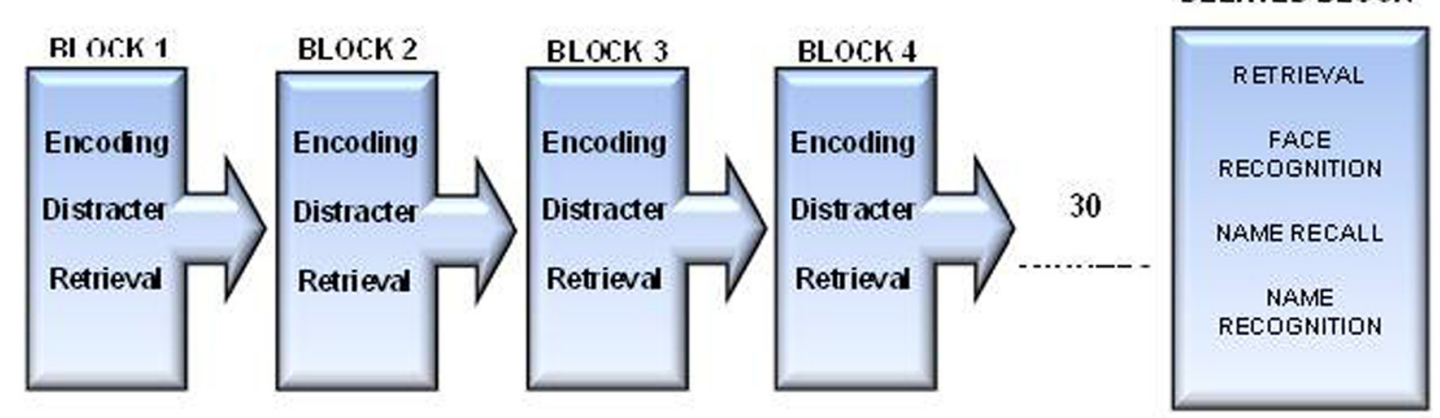

B

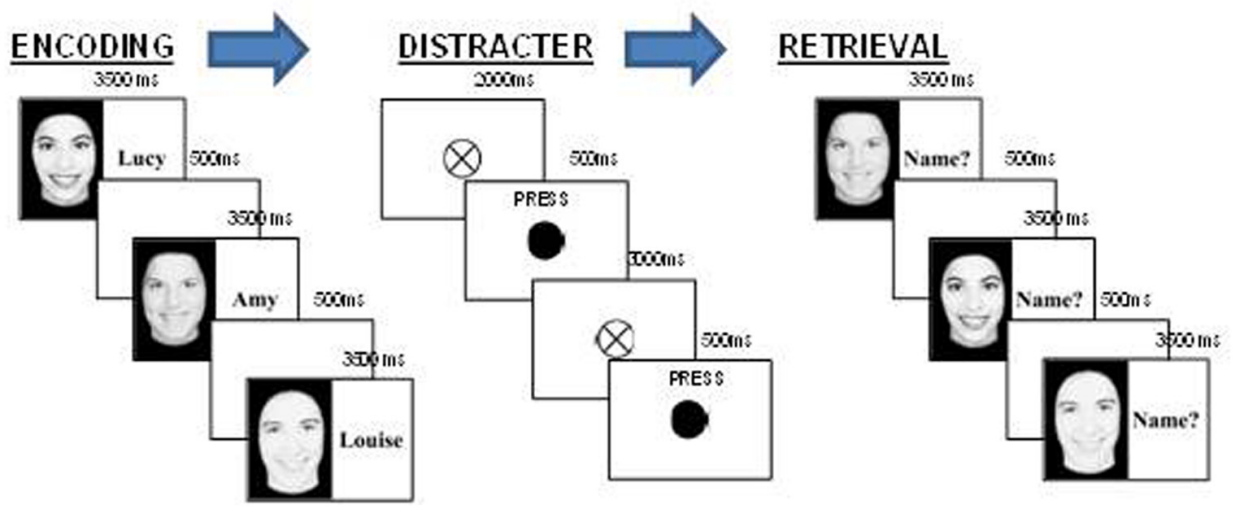

FIGURE 1 | (A) FNP running order. The Face-Name task comprises four blocks of encoding, distraction, and immediate retrieval followed by a block of delayed retrieval, which also involved name recall and face and name recognition components. (B) FNP; Encoding, distracter, and retrieval components. Participants first encoded the six faces and name for 3500 ms each, following this they carried out a distracter task where they were required to press the spacebar every time the fixation cross turned black. Each block ended with the retrieval component where they were asked to call out the name they believed belonged to each face. 
faces and names that were and were not previously seen (see Figure 1A). More details on each phase of the task are included below;

Face name encoding. During the encoding blocks, participants viewed six face-name pairs (each face was viewed once per block), which were presented serially at a rate of $3.5 \mathrm{~s}$ per pair, with an inter-stimulus interval of $500 \mathrm{~ms}$. The presentation order was constant across each of the encoding blocks.

Distracter Task. Between each encoding and retrieval block, a distracter task was presented to participants for $35 \mathrm{~s}$. During this task participants saw a fixation cross $(20 \mathrm{~mm})$ presented in the center of the screen, and at pseudo-random intervals of between 2 and $4 \mathrm{~s}$ the circle would turn black for $500 \mathrm{~ms}$. Participants were instructed to press the space-bar whenever the circle "flashed" black as quickly and accurately as they could. A total of 10 targets were presented to the participants.

Immediate face name retrieval. Following the distracter task participants viewed the six faces, which were presented in a randomized order, this time without the accompanying names. Each face was presented for $3.5 \mathrm{~s}$, with an inter-stimulus interval of $500 \mathrm{~ms}$, upon presentation of each face, participants were asked to vocally recall the name that they believed to correspond to each face. The experimenter recorded correct and incorrect responses, with a non-response being recorded as incorrect.

\section{Delayed face name retrieval}

After a 30-min delay, where participants were completing other tasks, participants were required to complete the recall as many correct face-name pairs as possible.

Delayed name recall. Following the $30 \mathrm{~min}$ delay, participants were asked to vocally recall as many names as they could remember from the experiment, the total names correctly recalled was summed and reported.

Delayed face and name recognition. Following the delayed Name Recall and delayed Face Name Retrieval, participants were instructed that they would be see fourteen faces on the screen, presented centrally. Each face remained on the screen until the participant chose to press the spacebar to move onto the next face. Six of the faces were those that had been seen in the experiment and the other eight were faces that had not previously been seen. For each face participants were instructed to circle "yes" or "no" on their response sheet, and then rate their certainty of their response by circling a number between 1 and 6 , where 1 was representative of them being highly confident of their response, and 6 being very unconfident of their response. They were then required to complete the same process for names. The total accuracy for this part of the experiment was the number of faces which were part of the experiment which were correctly identified as being part of the experiment. Misses were calculated as those faces which were part of the experiment, but were not identified by the participant. Correct foils were calculated as the total number of faces which were correctly identified as not being part of the experiment. False positives were calculated as those faces which were incorrectly identified as being part of the experiment.

The total certainty of responses was summed by calculating the percentage certainty for each response, and then adding together each of the fourteen percentage's together to create a total score.

Everyday memory questionnaire. This self-rating questionnaire (Sunderland et al., 1984) consists of twenty-eight questions which measure how often within the last 3 months participants believe they have had problems with aspects of memory which people should encounter on an everyday basis. The relative frequency of perceived memory problems are rated on a nine-point scale which ranges from "Not at all in the last 3 months" to "More than once a day," with higher scores indicative of more perceived problems with memory.

The total score was obtained by summing the individual scores (from 0 to 8 per question). The square root of the total score was then calculated (as in Sunderland et al., 1984), and is the score reported in this thesis.

\section{STATISTICS}

Results for this study were calculated using both repeatedmeasures and one-way ANOVA, with Bonferroni corrected post hoc comparisons being run where appropriate. Where the two groups were compared directly for one condition an independentgroups $t$-test was run, with adjusted degrees of freedom where appropriate.

\section{RESULTS}

When assessed using the EMQ Depressed patients rated themselves as having significantly more everyday memory problems than matched controls $[t(34)=3.71, p<0.01$; Control $5.47 \pm 0.5$; Depressed $8.08 \pm 0.5]$.

\section{RECALL PERFORMANCE}

When performance on the FNP test was measured betweengroups using a one-way ANOVA, there was a significant effect of group at learning block $1[F(1,35)=5.03, p<0.05]$, block $4[F(1,35)=7.19, p<0.05]$ and the delayed recall block $[F(1,35)=6.66, p<0.05$; see Figure 2A $]$.

When groups were assessed individually using a repeatedmeasures ANOVA, there was an overall effect of block on learning for the Control group $[F(4,64)=9.12, p<0.01]$. Post hoc tests showed a significant improvement in performance from block 1 $(M=1.77, \mathrm{SD}=0.4: 29.5 \%)$ to block $4(M=3.76, \mathrm{SD}=0.5$ : $62.7 \%)$ and also from block 1 to the delayed block $(M=3.24$, $\mathrm{SD}=0.4$ : 54.0\%; see Figure 2B).

When the Depressed group was assessed there was also a significant effect of block on performance $[F(4,72)=5.67, p<0.01]$. Post hoc tests showed that all significance was relative to performance in block $1(M=0.84, \mathrm{SD}=0.2: 14 \%)$ with improvements from this block been seen at block $2(M=1.58, \mathrm{SD}=0.3: 26.3 \%)$ block $3(M=1.9, \mathrm{SD}=0.3: 31.7 \%)$ block $4(M=2.16, \mathrm{SD}=0.3$ : $36.0 \%)$ and the delayed block $(M=1.95, \mathrm{SD}=0.3: 32.5 \%$; see Figure 2B).

When assessed for total learning over the first four blocks using an independent-samples $t$-test, there was a significant difference 


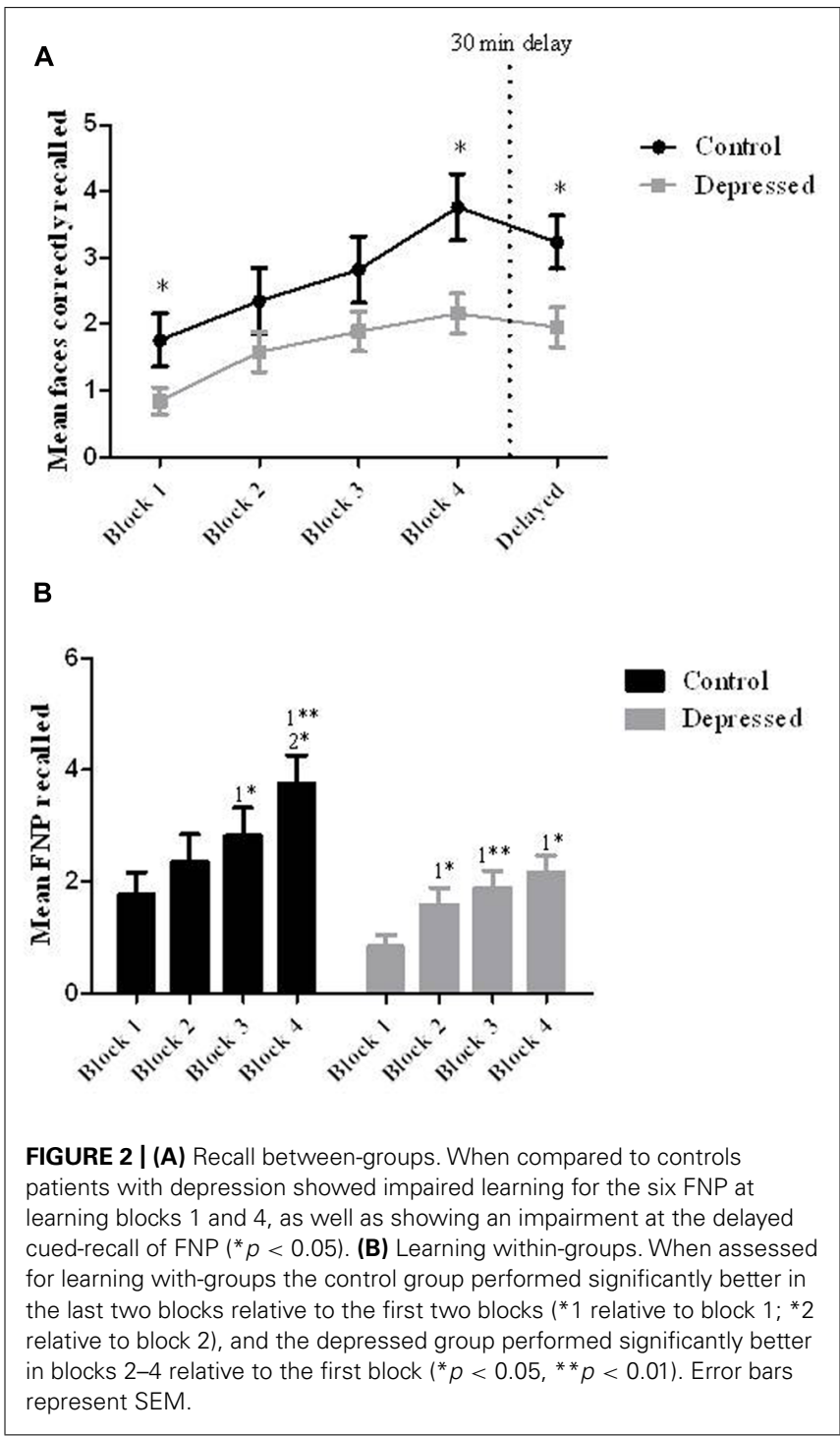

between the two groups $[t(25.6)=2.35, p<0.05]$, with the Depressed group recalling fewer faces than the Control group (Control: $10.71 \pm$ 1.61: 44.6\%; Depressed: $6.47 \pm 0.8$ : 30.0\%; see Figure 3A).

When the two groups were compared for delayed free recall of names for the task, there was a significant effect of group $[t(34)=2.43, p<0.05)$, with the Depressed group recalling significantly fewer names (Control: $5.06 \pm 0.2: 84.3 \%$; Depressed: $4.26 \pm$ 0.2: 71.0\%; see Figure 3B).

\section{RECOGNITION PERFORMANCE}

Despite there being a significant impact of Depression on the recall portion of the FNP task, there was no significant impact of Depression on any of the measures of recognition (see Figure 4), including the correct number of faces identified $[t(34)=0.85$, $P=0.4$; Control: $5.76 \pm 0.1$ : 96.0\%; Depressed: $5.63 \pm 0.1$ : $93.8 \%]$ or correct face foils identified $[t(34)=0.008, P=1$; Control: $7.53 \pm 0.3$ : 94.1\%; Depressed: $7.53 \pm 0.2$ : 94.1\%]. There was also no effect on the number of names correctly recognized

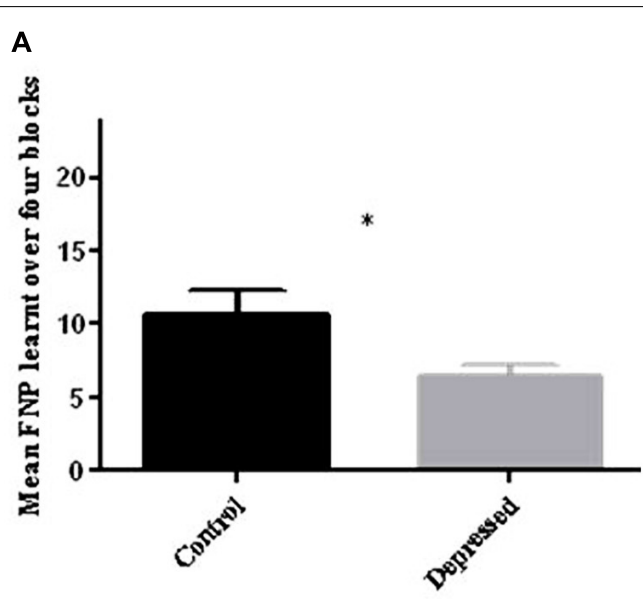

B

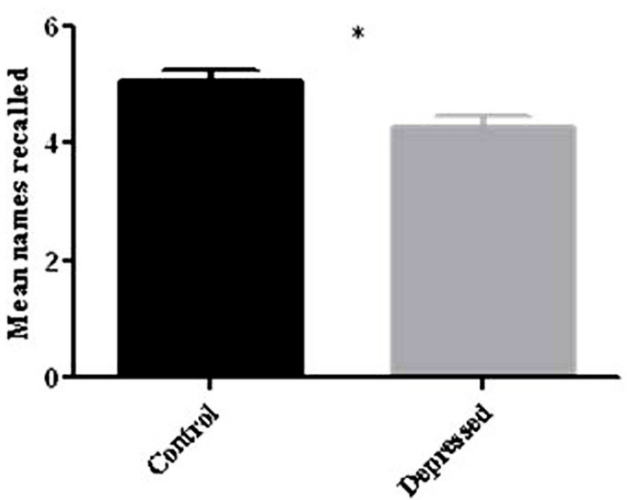

FIGURE 3 | (A) Total FNP recalled over four learning blocks. When compared to control participants depressed patients recalled significantly fewer FNP over the four learning blocks ( $\left.{ }^{*} p<0.05\right)$. (B) Free recall of names following half hour delay. When compared to control participants depressed patients recalled significantly fewer names after the half hour delay $\left({ }^{*} p<0.05\right)$. Error bars represent SEM.

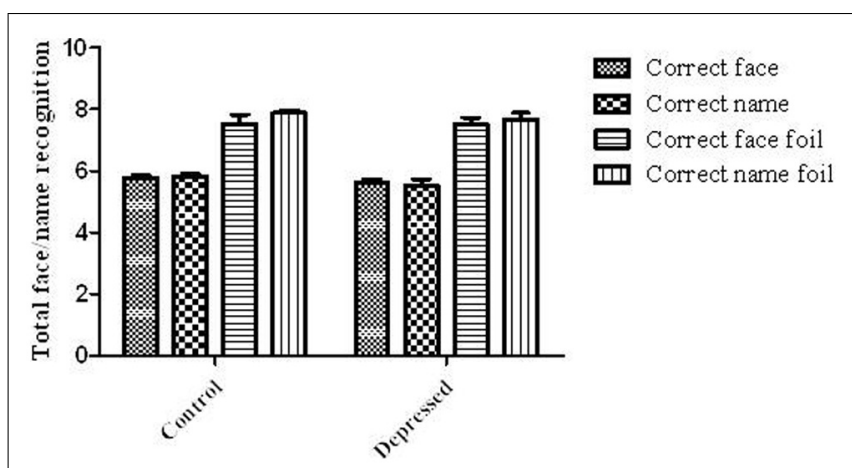

FIGURE 4 | Recognition of faces and names. There was no significant difference between the two groups for lures and foils for both the faces and names. Error bars represent SEM.

$[t(26.1)=1.38, P=0.2$; Control: $5.82 \pm 0.1$ : 97.0\%; Depressed: $5.53 \pm 0.2: 92.2 \%]$, or correct foils identified $[t(26.9)=1.14$, $P=0.3$; Control: $7.88 \pm 0.08$ : 98.5\%; Depressed: $7.68 \pm 0.2$ : $96.0 \%]$. 


\section{DISCUSSION}

Compared to age-sex and -education matched healthy controls depressed patients showed impairment in aspects of the FNP task related to associative learning, delayed cued-recall and delayed free-recall of names. This indicates that the face-name pairs task is sensitive to mneumonic impairment in a depressed patientpopulation.

When assessed within-groups over the four learning blocks, there was a significant increase in FNP recall relative to block 1 indicating that the Depressed group was learning over the course of the task. These responses however were at a significantly lower level than the Control group. This finding is supported by previous studies that show decreased performance of depressed patients in tasks such as the California verbal learning task (CVLT). This is believed to be due to impairment at the learning phase of the task (Elderkin-Thompson et al., 2006, 2007) as reflected by effects on immediate recall and the total rate of learning (Kizilbash et al., 2002). Other studies have also found that depressed patients are impaired in the early stages of memory tasks when encoding demands are high as well as in the delayed recall portion of a task (Brand et al., 1992). These studies support observations from this experiment where depressed patients showed a significant impairment at block 1 of the task as well as the delayed block. The impairment seen was specific to the recall and encoding portions of the experiment but not the recognition portion of the experiment, suggesting that Depressed patients are impaired in verbal-recall and associativerecall, but not recognition. This observation is supported by few studies (Calev and Erwin, 1985), with the majority of literature in this area finding that both recall and recognition are adversely affected by depression (MacQueen et al., 2002; Ramponi et al., 2004; Drakeford et al., 2010). However, this is possibly due to low number of target items and foils participants were required to identify. For example, Drakeford et al. (2010) found that depressed subjects showed impaired recollection but intact familiarity when subjects were required to identify 50 targets from a total of 100 items, with many other remember/know paradigms also using a large number of lures and foils (MacQueen et al., 2002).

However, it must also be acknowledged that alternative explanations also exist for the observed deficit in people with MDD. Both motivational and attentional deficits comprise some of the core symptoms of MDD, and it is possible that deficits in attention and/or motivation could be responsible for worsened recall but intact recognition (as recognition is less cognitively demanding). Some researchers hypothesize that problems with directing attention (Li et al., 2013) and task motivation (Scheurich et al., 2008) could be in part responsible for poorer performance in memory tasks within this population.

Results from this study should also be interpreted with caution due to the small sample size and various potentially biasing confounders which were not explicitly controlled for or investigated. Factors that could have an influence on cognitive performance in this population that were not explicitly investigated in this study include age, consumption of nicotine and the impact of potential sleep deprivation. Another important issue that could not be investigated or controlled for due to the small sample was examination of the subtype of depression, depression severity and length of depressive episode. However, it should be noted that participants in this study were hospitalized for their depression indicating that the depressive episode was significantly disabling. However, reviews of the neuropsychological functioning of depressed patients describe variable results regarding cognitive functioning in this population, and present evidence that depression severity is not necessarily related to performance (McClintock et al., 2010). Some researchers also suggest that the cognitive impairment in depression is non-specific and that the pathological symptoms of depression can be dissociated from neurocognitive symptoms (Reppermund et al., 2009).

Further methodological limitations of the study include the use of only female faces only in the task and the fact that there were not enough control participants to complete perfect matching with Depressed patients. However, there was no evidence from this study that there was a gender bias implicit in the task. Strengths of this study include the fact that, to our knowledge, this is the first investigation of the FNP task in people with depression. The FNP also has high ecological validity when compared to other memory tasks due to the use of faces and names.

While results from this task would suggest hippocampal dysfunction in depressed patients a functional imaging study needs to be conducted in this cohort using this task in order to elucidate the precise neuroanatomical structures involved in this deficit.

\section{CONCLUSION}

In conclusion there is evidence that Depressed patients do show learning over repeated blocks of the FNP associative memory task, but that this is at a significantly lower level than age-sex-andeducation matched Control participants. The depressed cohort studies here also showed impaired delayed recall (associative and verbal), however, there was a preservation of verbal and nonverbal recollection which is proposed to be due to the low number of targets and foils for this task.

\section{ACKNOWLEDGMENTS}

The authors would like to extend their thanks to all participants who took part in the study, as well as all staff at St. Patrick's Hospital. This research was funded by a 4-year Health Research Board grant.

\section{REFERENCES}

Baune, B. T., Miller, R., McAfoose, J., Johnson, M., Quirk, F., and Mitchell, D. (2010). The role of cognitive impairment in general functioning in major depression. Psychiatry Res. 176, 183-189. doi: 10.1016/j.psychres.2008.12.001

Brand, A. N., Jolles, J., de Wied, G. (1992). Recall and recognition memory deficits in depression. J. Affect. Disord. 25, 77-86. doi: 10.1016/0165-0327(92) 90095-N

Bremner, J. D., Vythilingam, M., Vermetten, E., Vaccarino, V., and Charney, D. S. (2004). Deficits in hippocampal and anterior cingulate functioning during verbal declarative memory encoding in midlife major depression. Am. J. Psychiatry 161, 637-645. doi: 10.1176/appi.ajp.161.4.637

Calev, A., and Erwin, P. G. (1985). Recall and recognition in depressives: use of matched tasks. Br. J. Clin. Psychol. 24(Pt 2), 127-128. doi: 10.1111/j.20448260.1985.tb01323.x

Chua, E. F., Schacter, D. L., Rand-Giovannetti, E., and Sperling, R. A. (2007). Evidence for a specific role of the anterior hippocampal region in successful associative encoding. Hippocampus 17, 1071-1080. doi: 10.1002/hipo.20340 
Drakeford, J. L., Edelstyn, N. M., Oyebode, F., Srivastava, S., Calthorpe, W. R. and Mukherjee, T. (2010). Recollection deficiencies in patients with major depressive disorder. Psychiatry Res. 175, 205-210. doi: 10.1016/j.psychres.2008.08.010

Elderkin-Thompson, V., Mintz, J., Haroon, E., Lavretsky, H., and Kumar, A. (2006). Executive dysfunction and memory in older patients with major and minor depression. Arch. Clin. Neuropsychol. 21, 669-676. doi: 10.1016/j.acn.2006. 05.011

Elderkin-Thompson, V., Mintz, J., Haroon, E., Lavretsky, H., and Kumar, A. (2007). Executive dysfunction and memory in older patients with major and minor depression. Arch. Clin. Neuropsychol. 22, 261-270. doi: 10.1016/j.acn. 2007.01.021

Frodl, T., Schaub, A., Banac, S., Charypar, M., Jager, M., Kummler, P., et al. (2006). Reduced hippocampal volume correlates with executive dysfunctioning in major depression. J. Psychiatry Neurosci. 31, 316-323.

Glahn, D. C., Robinson, J. L., Tordesillas-Gutierrez, D., Monkul, E. S., Holmes, M. K., Green, M. J., et al. (2010). Fronto-temporal dysregulation in asymptomatic bipolar I patients: a paired associate functional MRI study. Hum. Brain Mapp. 31, 1041-1051. doi: 10.1002/hbm.20918

Hammar, A., and Ardal, G. (2009). Cognitive functioning in major depression - a summary. Front. Hum. Neurosci. 3:26. doi: 10.3389/neuro.09.026.2009

Hickie, I., Naismith, S., Ward, P. B., Turner, K., Scott, E., Mitchell, P., et al. (2005). Reduced hippocampal volumes and memory loss in patients with early- and late-onset depression. Br. J. Psychiatry 186, 197-202. doi: 10.1192/bjp.186.3.197

Kizilbash, A. H., Vanderploeg, R. D., Curtis, G. (2002). The effects of depression and anxiety on memory performance. Arch. Clin. Neuropsych. 17, 57-67. doi: 10.1060/S 0887-6177(00)00101-3

Landro, N. I., Stiles, T. C., and Sletvold, H. (2001). Neuropsychological function in nonpsychotic unipolar major depression. Neuropsychiatry Neuropsychol. Behav. Neurol. 14, 233-240.

Li, Y. R., Weinborn, M., Loft, S., and Maybery, M. (2013). Patterns of prospective memory impairment among individuals with depression: the influence of cue type and delay interval. J. Int. Neuropsychol. Soc. 19, 718-722. doi: $10.1017 /$ S1355617713000180

Mackin, R. S., and Arean, P. A. (2009). Impaired financial capacity in late life depression is associated with cognitive performance on measures of executive functioning and attention. J. Int. Neuropsychol. Soc. 15, 793-798. doi: 10.1017/S1355617709990300

MacQueen, G. M., Galway, T. M., Hay, J., Young, L. T., and Joffe, R. T. (2002). Recollection memory deficits in patients with major depressive disorder predicted by past depressions but not current mood state or treatment status. Psychol. Med. 32, 251-258. doi: 10.1017/S0033291701004834

McClintock, S. M., Cullum, M., Husain, M. M., Rush, A. J., Knapp, R. G., Mueller, M., et al. (2010). Evaluation of the effects od severe depression on global cognitive function and memory. CNS Spectr. 15, 304-313.

Mervaala, E., Fohr, J., Kononen, M., Valkonen-Korhonen, M., Vainio, P., Partanen, K., et al. (2000). Quantitative MRI of the hippocampus and amygdala in severe depression. Psychol. Med. 30, 117-125. doi: 10.1017/S0033291799001567

Naismith, S. L., Hickie, I. B., Turner, K., Little, C. L., Winter, V., Ward, P. B., et al (2003). Neuropsychological performance in patients with depression is associated with clinical, etiological and genetic risk factors. J. Clin. Exp. Neuropsychol. 25, 866-877. doi: 10.1076/jcen.25.6.866.16472

Ramponi, C., Barnard, P. J., and Nimmo-Smith, I. (2004). Recollection deficits in dysphoric mood: an effect of schematic models and executive mode? Memory 12 , 655-670. doi: 10.1080/09658210344000189

Reischies, F. M., and Neu, P. (2000). Comorbidity of mild cognitive disorder and depression - a neuropsychological analysis. Eur. Arch. Psychiatry Clin. Neurosci. 250, 186-193. doi: 10.1007/s004060070023
Reppermund, S., Ising, M., Lucae, S., and Zihl, J. (2009). Cognitive impairment in unipolar depression is persistent and non-specific: further evidence for the final common pathway disorder hypothesis. Psychol Med. 39, 603-614. doi: 10.1017/S003329170800411X

Rogers, M. A., Kasai, K., Koji, M., Fukuda, R., Iwanami, A., Nakagome, K., et al. (2004). Executive and prefrontal dysfunction in unipolar depression: a review of neuropsychological and imaging evidence. Neurosci. Res. 50, 1-11. doi: 10.1016/j.neures.2004.05.003

Scheurich, A., Fellgiebel, A., Schermuly, I., Bauer, S., Wolfges, R., and Muller, M. J. (2008). Experimental evidence for a motivational origin of cognitive impairment in major depression. Psychol. Med. 38, 237-246. doi: $10.1017 /$ S0033291707002206

Sheline, Y. I., Sanghavi, M., Mintun, M. A., and Gado, M. H. (1999). Depression duration but not age predicts hippocampal volume loss in medically healthy women with recurrent major depression. J. Neurosci. 19, 5034-5043.

Sheline, Y. I., Wang, P. W., Gado, M. H., Csernansky, J. G., and Vannier, M. W. (1996). Hippocampal atrophy in recurrent major depression. Proc. Natl. Acad. Sci. U.S.A. 93, 3908-3913. doi: 10.1073/pnas.93.9.3908

Sperling, R., Chua, E., Cocchiarella, A., Rand-Giovannetti, E., Poldrack, R., Schacter, D. L., et al. (2003). Putting names to faces: successful encoding of associative memories activates the anterior hippocampal formation. Neuroimage 20, 1400 1410. doi: 10.1016/S1053-8119(03)00391-4

Sunderland, A., Harris, J. E., and Gleave, J. (1984). Memory failures in everyday life following severe head injury. J. Clin. Neuropsychol. 6, 127-142. doi: 10.1080/01688638408401204

van Paasschen, J., Clare, L., Yuen, K. S., Woods, R. T., Evans, S. J., Parkinson, C. H., et al. (2013). Cognitive rehabilitation changes memory-related brain activity in people with Alzheimer disease. Neurorehabil. Neural Rep. 27, 448-459. doi: $10.1177 / 1545968312471902$

Vythilingam, M., Vermetten, E., Anderson, G. M., Luckenbaugh, D., Anderson, E. R., Snow, J., et al. (2004). Hippocampal volume, memory, and cortisol status in major depressive disorder: effects of treatment. Biol. Psychiatry 56, 101-112. doi: 10.1016/j.biopsych.2004.04.002

World Health Organisation (2012). Depression Fact sheet no 396. Available at: http://www.who.int/mediacentre/factsheets/fs369/en/index.html

Zeineh, M. M., Engel, S. A., Thompson, P. M., and Bookheimer, S. Y. (2003). Dynamics of the hippocampus during encoding and retrieval of face-name pairs. Science 299, 577-580. doi: 10.1126/science.1077775

Conflict of Interest Statement: The authors declare that the research was conducted in the absence of any commercial or financial relationships that could be construed as a potential conflict of interest.

Received: 04 January 2013; accepted: 23 January 2014; published online: 12 February 2014.

Citation: Smith KJ, Mullally S, McLoughlin D and O'Mara S (2014) Validation of the face-name pairs task in major depression: impaired recall but not recognition. Front. Psychol. 5:92. doi: 10.3389/fpsyg.2014.00092

This article was submitted to Psychopathology, a section of the journal Frontiers in Psychology.

Copyright (C) 2014 Smith, Mullally, McLoughlin and O'Mara. This is an open-access article distributed under the terms of the Creative Commons Attribution License (CC BY). The use, distribution or reproduction in other forums is permitted, provided the original author(s) or licensor are credited and that the original publication in this journal is cited, in accordance with accepted academic practice. No use, distribution or reproduction is permitted which does not comply with these terms. 